\title{
Norois
}

Environnement, aménagement, société

223 | 2012

Villes petites et moyennes

\section{Les effets de la saisonnalité touristique sur l'emploi des moniteurs de sports nautiques dans le département des Landes}

Seasonal tourism effects on the jobs of sports monitors in Landes department

\section{Christophe Guibert}

\section{OpenEdition}

Journals

Édition électronique

URL : https://journals.openedition.org/norois/4213

DOI : $10.4000 /$ norois. 4213

ISBN : 978-2-7535-2043-1

ISSN : $1760-8546$

\section{Éditeur}

Presses universitaires de Rennes

\section{Édition imprimée}

Date de publication : 30 mars 2012

Pagination : 77-92

ISBN : 978-2-7535-2041-7

ISSN : 0029-182X

\section{Référence électronique}

Christophe Guibert, «Les effets de la saisonnalité touristique sur l'emploi des moniteurs de sports nautiques dans le département des Landes », Norois [En ligne], 223 | 2012, mis en ligne le 28 février 2014, consulté le 13 janvier 2022. URL : http://journals.openedition.org/norois/4213 ; DOI : https:// doi.org/10.4000/norois.4213 


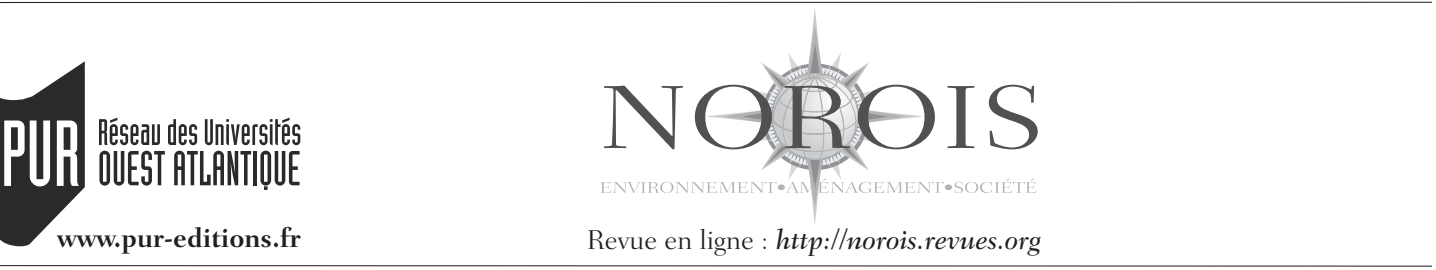

\title{
Les effets de la saisonnalité touristique sur l'emploi des moniteurs de sports nautiques dans le département des Landes
}

\author{
Seasonal Tourism Effects on the Jobs of Sports Monitors in Landes Department
}

\author{
Christophe GuiberT
}

ESO Angers - UMR CNRS 6590, Université d'Angers, UFR Ingénierie du Tourisme, du Bâtiment et des Services, 7 allée F.-Mitterrand, BP 40455 - 49004 AngERs Cedex 01 (christophe.guibert@univ-angers.fr)

\begin{abstract}
Résumé : Les activités nautiques constituent une offre de pratiques sportives importante au sein du littoral du département des Landes en période estivale. La variété des modalités de pratiques, la forte saisonnalité de la fréquentation touristique ainsi que des espaces de pratique spatialement limités font que le secteur professionnel des moniteurs diplômés d'État - dont la spécialité est de faire découvrir ou d'initier les activités nautiques à une clientèle très majoritairement estivale - est de plus en plus soumis à des processus de régulation quantitative émanant des collectivités locales. L'attractivité temporellement marquée du littoral landais et l'essor continu, ces dernières années, de l'enseignement des sports nautiques à finalité touristique produisent des effets sur les professions prises pour objet. Les profits symboliques et économiques engendrés par le département des Landes ne sont pas conformes aux avantages que peut espérer en attendre, professionnellement parlant, le secteur des moniteurs sportifs concernés.
\end{abstract}

\begin{abstract}
The nautical activities are an important offering of the Landes coast in summer. The variety of practice, the strong seasonality in tourist numbers and limited practice spaces make professional instructors - whose specialty is to discover or introduce water activities to customers overwhelmingly summer - is increasingly subjected to quantitative process control by local authorities. The attractiveness of the coastal Landes temporally marked and continuous rise in recent years of sports teaching produce effects on the profession. Symbolic and economic profits generated by the Landes are not conform to the benefits that can hope to expect, professionally speaking, the sports instructors sector.
\end{abstract}

Mots clé : Emploi sportif - saisonnalité touristique - concurrences spatiales - régulations politiques - Landes

Keywords: Sport Employment - tourism seasonality - spatial competition - political regulations - Landes

\section{INTRODUCTION}

Cet article vise à questionner les effets - inattendus - de la forte fréquentation touristique et des modes de régulations, à la fois balbutiants et itératifs, qui lui sont liés sur le secteur de l'emploi des moniteurs sportifs dans le département des Landes.
Le secteur des activités sportives et de loisirs est en effet une des filières touristiques « pour laquelle les réponses sont le moins satisfaisantes» (Siau, 2004). Les activités nautiques et de loisirs s'envisagent, pour les organismes de promotion touristique dans le département des Landes (Guibert, 2006), comme 
une spécificité forte dont les plus emblématiques sont le surf, le kite-surf, la plaisance, les sports motonautiques, le canoë-kayak, mais aussi la pêche. Une façade maritime longue de $106 \mathrm{~km}$ et la proximité avec la péninsule ibérique confortent la particularité de ce territoire. Une dizaine de lacs sont disséminés dans la zone rétro-littorale : à Hossegor, Seignosse, Soustons, Mimizan et Biscarosse pour les principaux d'entre eux sur lesquels se pratiquent des activités nautiques (voile, canoë-kayak, aviron, ski nautique). Les rivières importantes sont l'Adour, qui remonte de Bayonne vers Dax puis La Leyre, qui descend du Bassin d'Arcachon vers Morcenx, traversant ainsi du Nord au Sud le département. Le canoë-kayak et la pêche, notamment, sont largement présents sur ces cours d'eau.

La voile, le canoë-kayak et surtout le surf sont les activités nautiques les plus présentes dans l'offre de «tourisme sportif » (Sobry, 2004) au regard des stratégies des offices de tourisme et les plus pourvoyeuses en emplois de moniteurs sportifs (Chazaud, 2004; Guibert, 2002, 2006, 2008 ; Duceux, 2008). Le nombre d'emplois dans les écoles (associations et entreprises) est très élevé mais les postes sont fortement dépendants, pour la plupart d'entre eux, de la saisonnalité estivale, elle-même calquée sur les périodes touristiques des territoires (Guibert, 2010). Les spécificités des activités nautiques à finalité touristiques dans le département des Landes font que les effets de la saisonnalité, à laquelle sont liés les plus forts taux de fréquentation, structurent temporellement les métiers de moniteurs sportifs (codes NAF 92.6 A et C). Il en résulte qu'un grand nombre d'entre eux se trouve inemployés hors saison, principalement ceux qui encadrent les activités telles que le surf, la voile et le canoë-kayak dont la découverte et l'initiation par les pratiquants se réalisent principalement en juillet et août. L'installation dans une forme d'instabilité professionnelle potentielle - et les sentiments d'insécurité qui lui sont liés (Castel, 1995; Paugam, 2001, 2009) - dépend en partie du travail aléatoire, des trajectoires erratiques ou encore de périodes récurrentes de chômage. Aussi, le temps étant compté et les espaces de pratique spatialement limités, quels sont les effets de la forte fréquentation des lieux de pratiques d'activités nautiques sur l'emploi des moniteurs - c'est-à-dire le «noyau dur » de l'emploi sportif (Le Roux, 2003) -, en particulier ceux ayant en charge l'enseignement de la pratique du surf? Quels sont, en guise de réponses, les recours des élus politiques locaux pour limiter les fréquentations littorales et les concurrences d'une part puis pour encadrer les multiples usages du littoral landais d'autre part?

L'alternance des saisons est marquée par une économie touristique calme l'hiver et très dense l'été, des modalités de pratiques licenciées, plutôt compétitives avec un encadrement technique de type brevet d'État d'éducateur sportif (BEES) l'hiver et à l'inverse de nombreux pratiquants non licenciés, peu compétiteurs, encadrés par des moniteurs titulaires d'un brevet professionnel de la jeunesse de l'éducation populaire et du sport (BPJEPS) l'été. Plus que des questions migratoires liées à ces deux temporalités, toujours difficilement chiffrables dans les secteurs les moins porteurs économiquement, il s'agit de montrer que les spécificités des activités nautiques, à plus ou moins fortes dimensions touristiques, auxquelles sont liés les plus forts taux de fréquentation, structurent temporellement les métiers d'éducateur et de moniteur sportif selon les saisons.

À l'aune d'un ancrage théorique mobilisant des références croisées en sociologie et en géographie, il convient dans un premier temps d'identifier les variables qui positionnent le territoire concerné comme lieu privilégié de «tourisme littoral». Seront ensuite analysées les conditions explicitant les concentrations territoriales et les fortes densités de pratiquants d'activités nautiques en période estivale. Enfin, à partir de ces particularités spatialement localisées et des politiques municipales régulatrices, les répercussions sur le secteur d'emplois des moniteurs sportif seront évaluées. Cet article vise ainsi à contribuer à l'analyse de la dimension spatiale des sociétés - dans le domaine des pratiques sportives touristiques littorales - en insistant sur les modes d'actions, c'est-à-dire les pratiques sociales des espaces d'une part et les régulations, c'est-à-dire sur les politiques territorialisées d'autre part.

\section{Cadrage méthodologique}

Les résultats et les analyses présentées dans cet article proviennent de données croisées, collectées dans le cadre de deux rapports d'études principalement. Le premier intitulé Le surf en France: Emplois, employabilité et formations, a été comman- 
dité par la DRDJS Aquitaine-Gironde en 2008. Le second, Etat des lieux des activités nautiques et de loisirs touristiques dans le département des Landes, a été réalisé à la demande du CREPS d'Aquitaine, la DRJS d'Aquitaine et la DDJS des Landes en 2010.

Des recherches documentaires d'institutions et d'observatoires (INSEE, Pôle Emploi, Chambre de commerce et d'industrie des Landes, Comité régional de tourisme d'Aquitaine et Comité départemental de tourisme des Landes) ont complété les exploitations de données statistiques disponibles auprès du «mouvement sportif » (Comités sportifs départementaux, fédérations, Jeunesse et Sports). Des entretiens (avec des moniteurs, des dirigeants d'associations et d'entreprises, des responsables d'offices de tourisme et des élus locaux) ont par ailleurs permis de mesurer plus finement les caractéristiques des lieux de travail et des situations professionnelles des moniteurs sportifs. Ainsi, cadrées statistiquement, les observations de terrain, de type monographique, ont fait l'objet d'un travail minutieux visant à décrire les lieux de pratique, les installations et infrastructures offertes aux membres et/ou aux clients, en illustrant nombre d'explications par des photographies des sites visités. Si les communes de Biscarosse, Soustons et Capbreton (pour leurs offres variées d'activités nautiques) ont été privilégiées dans le cadre de monographies, l'ensemble des communes littorales a toutefois été pris en compte.

\section{LES LANDES : UN TERRITOIRE TOURISTIQUE À LA SAISONNALITÉ STRUCTURANTE}

En 2005, le tourisme a généré 48600 emplois salariés en Aquitaine, majoritairement des emplois saisonniers à durée déterminée. L'emploi salarié touristique double entre janvier et août en Aquitaine. Il triple même dans les hébergements touristiques non hôteliers, les campings et les centres de vacances. Quels qu'ils soient, ces emplois sont directement liés à la fréquentation touristique qui peut être mesurée grâce au nombre de nuitées comptabilisées : un million de touristes est ainsi dénombré les jours de pointe en août. Globalement, plus d'un touriste sur deux $(51 \%)$ vient en Aquitaine pendant les mois de juillet et août et près des trois quart entre mai et septembre selon le Comité régional de tourisme de la région (CRT, 2005).
Première destination touristique d'Aquitaine, le département des Landes est la destination choisie par deux millions de touristes chaque année dont 200000 étrangers (principalement des Espagnols, des Anglais, des Allemands et des Néerlandais). Le littoral et les activités qui lui sont ordinairement liées, la forêt mais aussi le thermalisme, le patrimoine culturel et archéologique puis la gastronomie sont les principaux « atouts » des Landes. Les manifestations et les animations culturelles, musicales et sportives sont également importantes en termes d'impacts touristiques et de notoriété territoriale. C'est notamment le cas pour le "Quiksilver Pro », une compétition professionnelle de surf dans le sud du littoral landais, fréquenté à chaque édition, fin septembre-début octobre, par 25000 spectateurs. Le secteur d'activité « Tourisme et Loisirs », selon le classement opéré par les Assedic en 2007, compte 5541 emplois soit une hausse de 34,1\% par rapport aux données répertoriées en 2000. Ce secteur est un de ceux dont la croissance, en 7 ans, a été la plus remarquable: seuls les «Services financiers et immobiliers » : $+46 \%$ et les « Services au entreprises » : $+43 \%$ ont une croissance supérieure. À l'échelle nationale, le secteur du « Tourisme et Loisirs » a vu croître ses effectifs de 17,8 \% entre 2000 et 2007 , ce qui témoigne d'une cristallisation forte de ce secteur d'activités dans le département des Landes avec une hausse deux fois supérieure à la moyenne nationale (INSEE, 2009). Toutefois, selon le CDT des Landes, 21 millions de nuitées ont été comptabilisées en 2007 dont $87 \%$ entre avril et septembre et, surtout, $62 \%$ en juillet août (avec un pic mi-août), ce qui témoigne d'une saisonnalité forte aux effets structurants sur l'offre d'activités nautiques à vocation touristique d'une part et sur l'emploi - saisonnier - du secteur d'autre part (CDT des Landes, 2007).

Si le climat en période automnale et hivernale n'est pas des plus propice à la découverte et à l'initiation des activités nautiques dans leur ensemble, force est de constater que cela ne constitue pas l'unique obstacle au maintien de la fréquentation touristique. Les municipalités profitent en effet de ces périodes de répit pour aménager, réviser et restaurer les sites. La ville de Capbreton opère ainsi à un rechargement artificiel de sable à l'endroit des plages situées au sud de l'embouchure du port. Au-delà de cette spécificité littorale (Capbreton est 
l'unique port des Landes), les réaménagements et embellissements des fronts de mer sont effectués en automne et hiver, ce qui n'implique pas un maintien, ne serait-ce que partiel d'une fréquentation touristique annualisée (figures 1 à 4). Il en résulte une fermeture quasi-complète des magasins destinés davantage à une clientèle estivale qu'aux résidants annuels (prêt-à-porter, bars et restaurants, surfshops) et la mise en veille annualisée des produits touristiques portés vers les loisirs nautiques.

L'offre touristique du département répond donc à des contraintes temporelles fortes avec de nettes dissociations entre les saisons en termes de fréquentation. Les offres d'activités nautiques touristiques, plus particulièrement, sont en conséquence étroitement liées à ces singularités littorales. Toutefois, en été, ces activités contribuent amplement à dynamiser l'attraction touristique du département de Landes comme en témoignent les stratégies des collectivités locales.
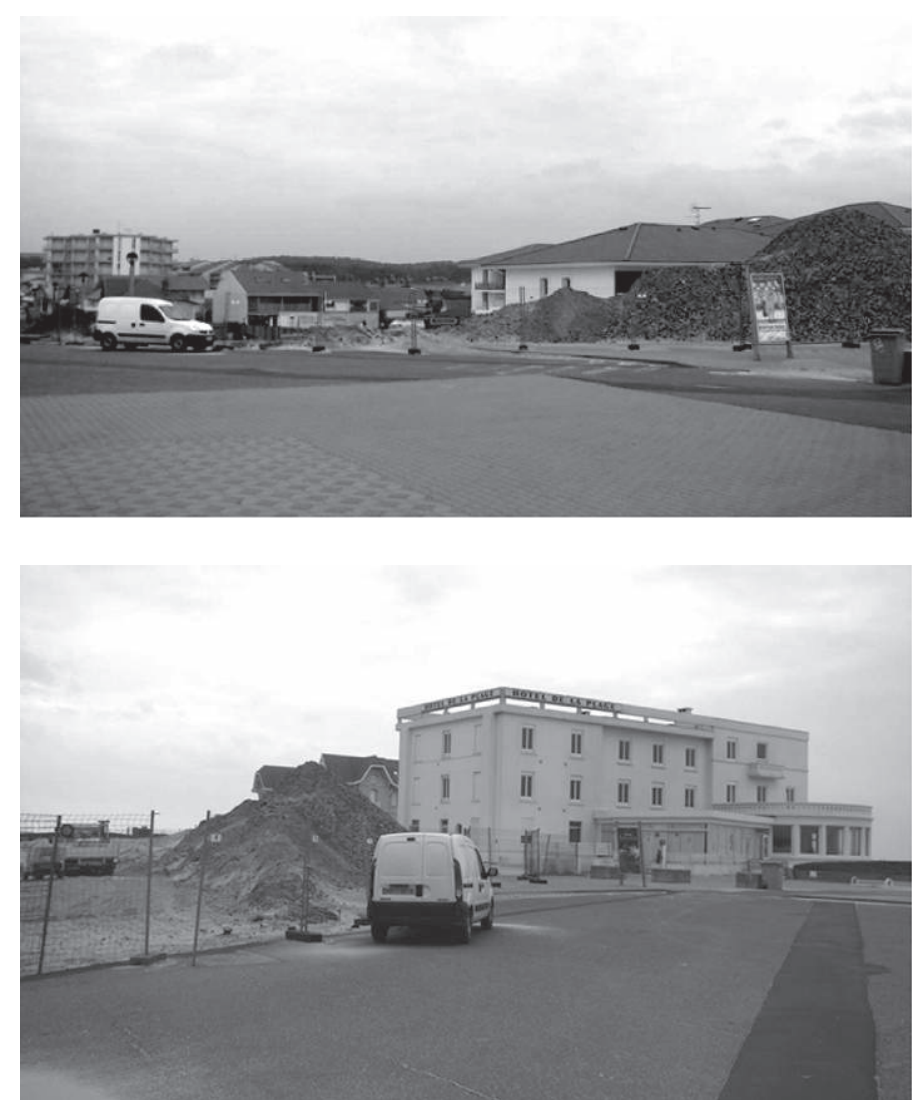

\section{LES ACTIVITÉS NAUTIQUES AU PROFIT DU DYNAMISME TOURISTIQUE DU TERRITOIRE}

\section{«La glisse tient le haut du palmarès »}

Les activités nautiques et de loisirs sont une spécificité forte du département des Landes. La croissance des fréquentations et des aménagements des espaces de pratique, les transformations technologiques des matériels utilisés, l'appropriation de sites et itinéraires «sauvages », les impacts de ces activités sur l'économie locale - et plus largement sur le tourisme -, puis sur l'emploi sportif sont la marque d'un développement territorial dynamique. En effet, a fortiori dans un département touristique comme celui des Landes, sports nautiques et tourisme ne peuvent plus être appréhendés tels des domaines séparés, tant dans les usages que dans les analyses. Comme l'atteste l'étude menée par le Comité Départemental du Tourisme des Landes de juin à septembre 2003,
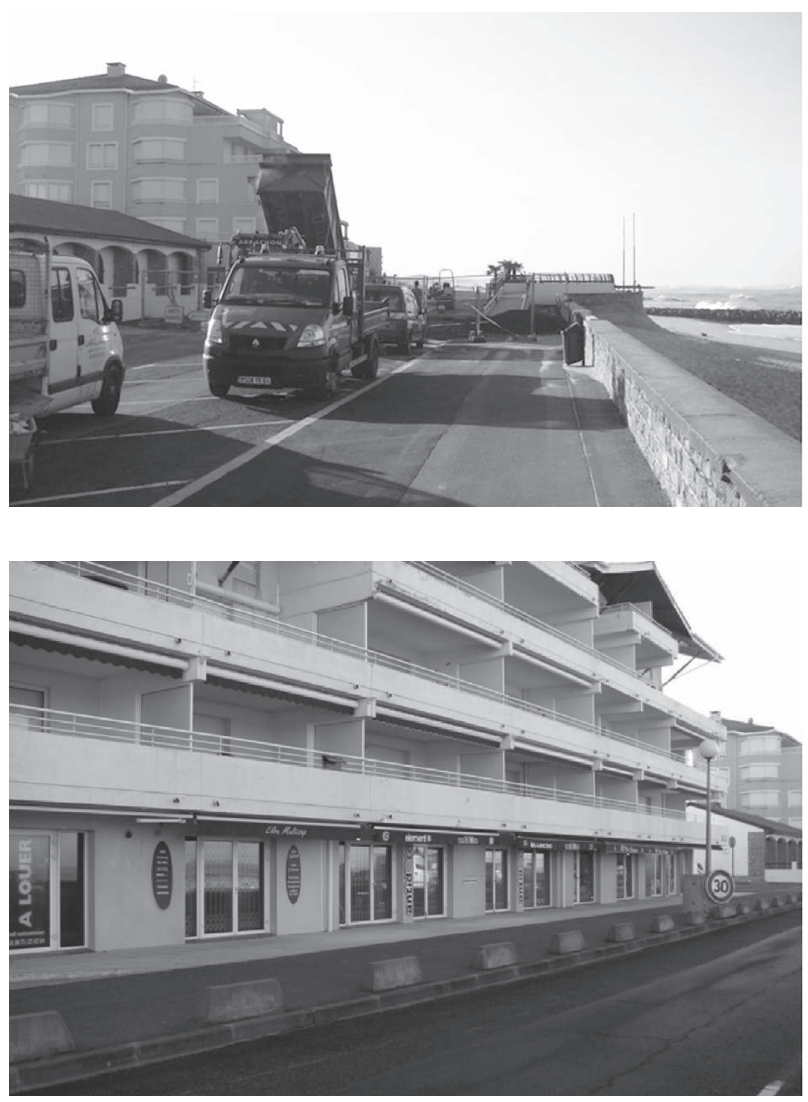

Figures 1, 2, 3 et 4: Une activité touristique réduite dès l'automne

Travaux sur le front de mer à Biscarrosse (colonne de gauche).

Travaux sur le remblai et activité économique réduite (magasins du front de mer) à Capbreton (colonne de droite).

A reduced tourism in autumn 
les retombées touristiques sont directement perceptibles. À partir d'une question à choix multiples proposée à un échantillon de 3000 touristes des stations balnéaires du département (CDT des Landes, 2003), la pratique de la "glisse », au sens large du terme, se situe au quatrième rang des "activités préférées » des personnes interrogées derrière « la plage » $(88,6 \%)$, « les promenades à pied » $(44,8 \%)$ et «à vélo» $(40,4 \%)$. Plus de $11 \%$ des individus questionnés disent venir en vacances sur ce littoral pour la "glisse », loin devant d'autres activités comme « le golf » $(2,6 \%)$ ou la « rando vélo » $(4,3 \%)$. Plus récemment, l'enquête clientèle du CDT menée en 2008 (3350 enquêtés de juin à septembre) consolide l'analyse. Les «motivations » des touristes à la question relative au choix de la destination touristique est sans équivoque : 76,6\% des clients déclarent venir dans les Landes pour « les plages et les vagues » et, « parmi les activités sportives, la glisse garde sa place en haut du palmarès » (CDT des Landes, 2008). Le surf matérialise ainsi un processus puissant d'identification territoriale.

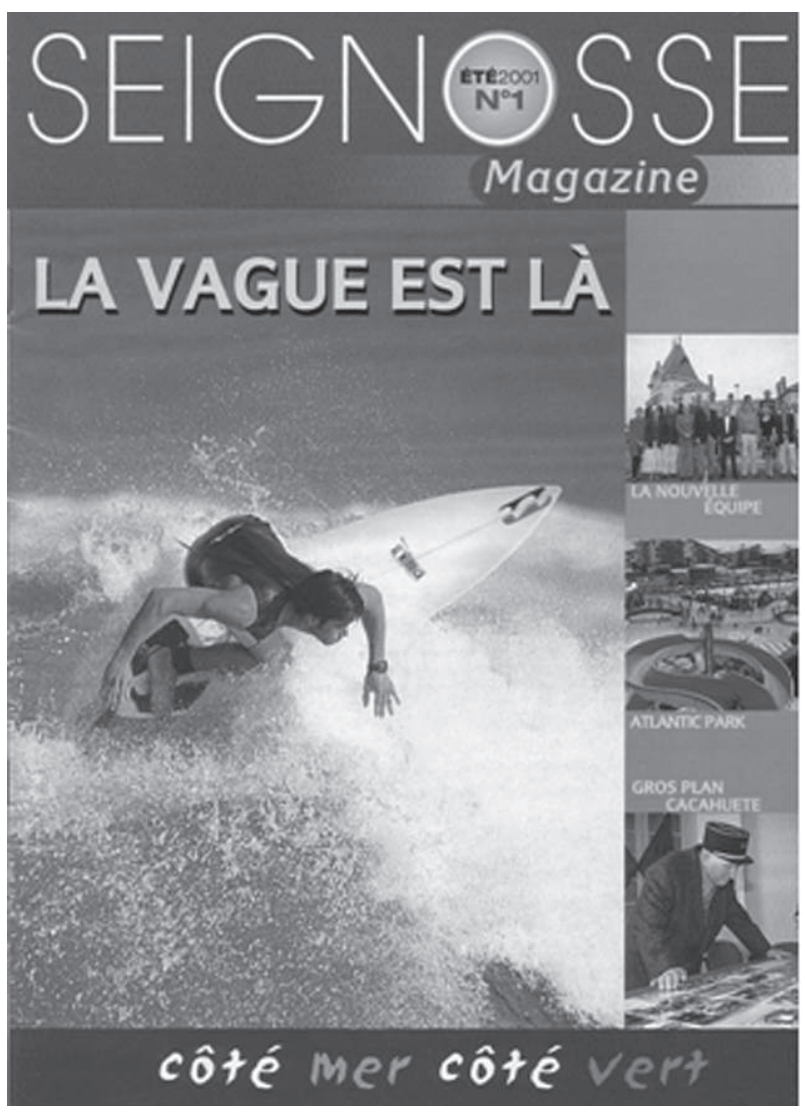

\section{Le surf : une activité articulant l'offre sportive touristique}

Avec une histoire du surf - initialement localisée à Biarritz - qui remonte à la fin des années 1950, une « vie fédérale » dynamique (le siège de la Fédération Française de Surf est situé à Hossegor depuis 1964), une localisation des entreprises du surfwear (présence des sièges sociaux européens des entreprises Rip Curl, Billabong, etc.) et une «qualité » de vague reconnue dans les médias spécialisés, les Landes sont sans conteste un des principaux départements de surf français. À ce titre, J.-J. et D. Bavoux (1998) précisent que «l'engouement est très fort pour les activités sportives dites de glisse. Grâce au développement de ces activités, des côtes jusqu'alors défavorisées à cause de leurs rochers, de leurs vents vigoureux ou de leurs vagues puissantes peuvent connaître un succès inespéré. » Le mot d'ordre, depuis quelques années déjà (Augustin, 1994), est de mettre en avant la qualité des vagues ou les différents spots de $\operatorname{surf}^{1}$ : la rhétorique des slogans et publicités des communes et offices de tourisme affi-

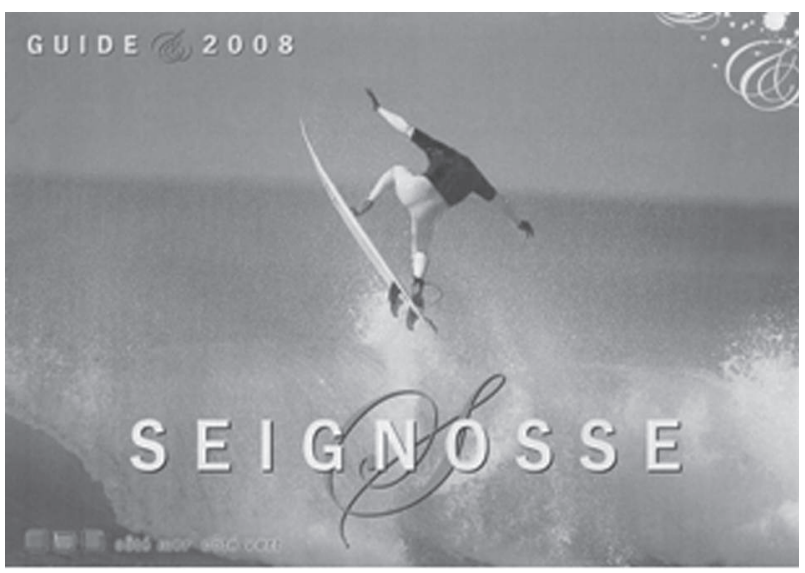

Figures 5 et 6 : «La vague est là » : la communication proclamée d'une commune en direction du surf, Eté 2001 et Couverture du Guide 2008 Si « la vague est là », c'est qu'elle n'est pas ailleurs. Ce slogan positionne Seignosse, selon la volonté de la municipalité, comme le spot incontournable en France.

"The wave is there": a municipality proclaimed communication in the direction of surfing

1. Le cas révélateur de la ville d'Hendaye près de Bayonne montre qu'il n'en a pas toujours été ainsi. En 1960, un dépliant touristique de la station balnéaire proposait, outre un nombre important d'activités physiques ou culturelles, une "immense plage de sable fin en pente douce sans aucun danger . 
chent le «plaisir » et les «sensations » que peuvent potentiellement apporter les vagues et le surf. Ce processus d'attraction par les vagues, et de manière générale par les sports apparentés (surf, bodyboard, longboard) utilisant ce support, est assuré par les ressources intrinsèques du littoral landais.

Des communes mobilisent la carte du surf à l'aide de slogans évocateurs et orientés comme, dans les autres départements aquitains, Biarritz, "La capitale européenne du surf " (Marchais, 1994) ou encore Lacanau, " La ville du surf ». La communication de certaines équipes municipales et offices de tourisme atteste cette volonté de développer un tourisme porté sur le surf (Guibert, 2006), à l'image de Seignosse dont la couverture du premier numéro du magazine municipal en 2001 - « La vague est là »-est on ne peut plus explicite (figures 5 et 6), tout comme la couverture du Guide 2008 pour la saison estivale :

Les responsables des offices de tourisme de Capbreton, Soustons et Biscarrosse constatent unanimement que le surf occupe une position prédominante dans l'espace des sports nautiques de chaque commune. Demande de la clientèle en vacances, stratégies de communication et de «marketing territorial » puis impacts économiques et sociaux positionnent le surf comme un véritable atout de promotion. Si cette orientation est pleinement assurée à Capbreton, les offices de tourisme de Soustons et Biscarrosse complètent cette « image surf » à l'aide d'une offre sportive variée, notamment grâce aux lacs. Les spécificités locales (offre d'activités nautiques, situation géographique, histoire touristique, etc.) font que les offices de tourisme adaptent leurs politiques touristiques et leurs stratégies de communication (ville sportive avec le Centre Européen de Rééducation du Sportif (CERS) à Capbreton; ville «multisports » et non uniquement surf à Biscarrosse; la baignade, le surf et un projet de «vague artificielle » à Soustons). Toutefois, les activités nautiques - et en particulier le surf constituent bien, au-delà de l'essor global sur le plan quantitatif, une offre sur laquelle chaque office de tourisme centre son action :

Directrice de l'office de tourisme de Capbreton ${ }^{2}$ : "Le surf, ça a une très bonne image »

2. Entretien effectué auprès de la Directrice de l'office de tourisme de Capbreton, août 2009.
«D'abord le surf, c'est le plus important: ça concerne $70 \%$ à $80 \%$ de la demande des touristes qui viennent ici au bureau. Il y a de plus en plus de demandes pour aller dans une école de surf. Et quand on discute avec chaque responsable d'école, ils disent qu'il y a du boulot pour tout le monde! On sent que le surf ça a une très très bonne image et ça fait partie de la notoriété des Landes, pas seulement de Capbreton. Ensuite ça concerne le jet ski, le kite surf alors que paradoxalement nous n'avons pas d'association de kite. On a une demande surtout en saison pour le kite surf donc on les envoie sur Biscarrosse. Il y a la voile, les sorties en mer avec des entreprises, des associations puis sur les lacs de Soustons ou Hossegor. Il y a aussi le sawvetage côtier qui se développe donc tout ce qui a attrait aux sports d'eau, c'est important. La demande est bien assouvie et on a une offre assez large."

"Les vagues, elles sont ici! C'est sûr qu'il y en a qui font des kilomètres pour venir dans les bonnes vagues. Ici, c'est assez exceptionnel: les frontières ne s'arrêtent pas... il faut que la vague soit bonne! Justement, sur nos dépliants, on communique sur cette belle image parce que le surf, ça veut dire: environnement, bonne santé, jeunesse, sport... On veut essayer de rester une station jeune, dynamique, sportive et donc le surf ça donne une bonne image. »

Directrice de l'office de tourisme de Biscarrosse $^{3}$ : «Le surf est présent dans les mentalités des gens. »

"La qualité de l'espace naturel: nous on communique là-dessus. Mais ce qui fait la différence: c'est qu'on a trois pôle: Biscarrosse-ville, Biscarrosselac et Biscarrosse-plage, mais ça en fait aussi une difficulté parce que les touristes doivent faire des kilomètres pour aller d'un point à un autre. Par contre l'avantage, c'est qu'on des pôles très typés. Biscarrosse-plage, c'est 2000 habitants à l'année mais 50000 à 60000 l'été! [...] On a des sites superbes: on peut pratiquer tout les activités que l'on veut! Nous, notre activité à l'office de tourisme, elle est plus basée sur tout ce qui est surf. Il y a un engouement fou pour le surf. Et depuis quatrelcinq ans, la demande auprès des écoles de surf, elle commence dès les mois de mars et avril. [...] Le surf est

3. Entretien effectué auprès de la Directrice de l'office de tourisme de Biscarrosse, novembre 2009. 
présent dans les mentalités des gens, pas tous, mais c'est présent. »

Directrice de l'office de tourisme de Soustons ${ }^{4}$ : "On bénéficie de l'image des communes d'à côté ${ }^{5}$." "Les sports nautiques, c'est ce qu'on met en avant, c'est ce qu'on vend. Vous avez l'océan, vous avez cinq lacs et des rivières: donc l'eau est omniprésente. Et aujourd'hui, tous les sports se sont développés : on peut tout faire sauf du ski nantique. On peut faire de la voile: pour les touts petits et pour les plus grands. La descente de rivière : il y a le canoë kayak ou avec des barques. Et ensuite il y a toute la déclinaison du surf : donc surf, bodyboard, etc. Et on peut même faire du jet ski. Il y a aussi le paddle board... mais alors là, il va y avoir des problèmes de cohabitation avec la voile, le canoë, la pêche, etc. sur les lacs et sur le courant. La demande augmente aussi en kite surf: on a de plus en plus de demandes. Il y a bien une demande par jour en saison estivale pour savoir où pratiquer. "

"Cette offre très variée est un atout et un frein également parce qu'il faut rester cohérent. La nouvelle équipe municipale est très axée sur Soustons Plage. Donc ce qu'on met en avant en premier c'est la baignade et le surf parce qu'il y a quand même Hossegor, Biarritz... on profite de la renommée, il ne faut pas se leurrer! Mais il y a quand même un gros projet autour du surf à Soustons Plage. Il y a un projet de vague artificielle à Soustons Plage puis un centre de haut niveau en surf 6 ... Garder quelque chose comme ça sur place, ce serait très intéressant! Donc il y des discussions au sujet de la vague artificielle. Il y a très peu d'endroits au monde où il y a des vagues artificielles. »

Les impacts touristiques et économiques des activités nautiques sur le littoral landais en été sont bien établis et reconnus par nombre d'études menées par les chambres consulaires locales notamment. Les organismes locaux en charge de la promotion

4. Entretien effectué auprès de la Directrice de l'office de tourisme de Soustons, septembre 2009 .

5. N'ayant pas les attributs spécifiques donnés et construits nécessaires, Soustons peut jouer le principe, sur le plan symbolique, de la « rente de qualité territoriale » (Mollard, Pecqueur, 2003).

6. Majoritairement financées par le Conseil Général des Landes (à hauteur de $56 \%$ ), les nouvelles installations fédérales (dénommées « académie du surf et des activités du littoral ») ont été inaugurées début 2010 en présence d'Henri Emmanuelli, président du Conseil général. D’une surface de $400 \mathrm{~m}^{2}$ (salles de réunion, bureaux), le Comité départemental des Landes de surf y est hébergé. des territoires, comme en témoignent les extraits d'entretiens ci-dessus, mobilisent ainsi largement ces pratiques sportives estivales à destination d'une clientèle touristique. Toutefois, à l'aune de la temporalité de la fréquentation touristique littorale, les effets concernant les caractéristiques des métiers de moniteurs sportifs sont directs, notamment au sujet de la saisonnalité de la profession.

\section{LA SAISONNALITÉ DES MÉTIERS D'ÉDUCATEURS SPORTIFS D'ACTIVITÉS NAUTIQUES DANS LES LANDES}

Les caractéristiques des activités nautiques dans le territoire concerné renforcent l'idée selon laquelle il existe une sorte de cristallisation spatiale autour de certaines communes littorales d'une part, puis une concentration professionnelle propre à une activité en particulier, le surf.

\section{Typologie et répartition des établissements employeurs en 2009 dans le domaine des activités nautiques : une double agrégation}

Pour le compte de l'année 2009, selon les données statistiques de la Direction Départementale de la Jeunesse et des Sports des Landes (nouvellement "intégrée » au sein de la Direction Départementale de la Cohésion Sociale et de la Protection des Populations), le nombre d'établissements employeurs dans le domaine des activités nautiques représente environ un quart de la totalité des établissements employeurs dans le sport et les activités physiques (164 sur 682, soit 24,05\%). Concernant les disciplines pourvues en éducateurs rémunérés, le surf occupe une position prédominante sur le plan quantitatif. Ainsi, à partir d'une extraction statistique plus fine, 99 associations et entreprises de prestations de surf sont employeurs dans le département, soit près de 14,5\% de l'ensemble des sports tous confondus mais plus de $60 \%$ des activités nautiques (soit trois établissements employeurs sur cinq), renforçant dès lors les effets de concentration. Suivent, sur le plan quantitatif, le canoë (30 établissements), la voile (20 établissements), le sauvetage et secourisme (4 établissements), la pêche et l'aviron (respectivement 2 établissements) puis le jet ski (1 établissement). 
Toutefois, tous les établissements employeurs ne sont pas centrés sur une seule activité nautique. Les clubs et les entreprises de voile et de canoë-kayak sont, plus souvent que les autres activités nautiques, prestataires de «multi activités».

La répartition des associations et des entreprises qui emploient des moniteurs sportifs dans le domaine des activités nautiques est étroitement liée aux territoires les plus touristiques du département. Ces activités sont majoritairement envisagées par les responsables de clubs et d'entreprises dans des logiques de découverte et d'initiation à l'égard d'une clientèle touristique. Indépendante des principaux bassins de population du département (Mont-deMarsan et Dax notamment), la concentration - ou l'agrégation - des établissements employeurs est également fonction de l'existence de sites et d'espaces de pratique. Les écoles sont réparties au sein de 48 communes, sur les 331 du département des Landes. Sur les 164 établissements recensés dans le domaine des sports nautiques, 110 sont situées dans des communes littorales (par exemple : 21 à Seignosse, 18 à Biscarrosse, 13 à Mimizan, 10 à Hossegor, 8 à Vieux-Boucau, 7 à Capbreton et Messanges, 5 à Moliets et Léon, etc.), soit les deux tiers, et la majorité restante dans les communes rétro-littorales telles que Angresse, Sanguinet, Tosse, etc.

\section{Les spécificités temporelles du métier de moniteur sportif}

Dans la région Aquitaine, la saisonnalité touristique est, on l'a dit, la plus fortement marquée dans les Landes. Au sein du département, le nombre d'emplois touristiques salariés triple entre janvier et août : sur les 10400 emplois supplémentaires à l'année, $58 \%$ concernent les effectifs des mois d'été, soit 6000 emplois environ. Les effets de la saisonnalité touristique empêchent, quasi-mécaniquement, de développer une activité professionnelle annualisée liée à la découverte et l'initiation des sports nautiques. Par exemple, $71 \%$ de l'ensemble des emplois accompagnés par le groupement d'employeurs Sport Landes en 2009 étaient des CDD saisonniers. L'enquête du Comité départemental de tourisme des Landes de 2006 souligne également que la fréquentation des clientèles des clubs et des entreprises offrant des services de découverte et d'initiation d'activités nautiques s'élèvent à $70 \%$ de la fréquentation totale pour les mois de juillet et août, puis à $93 \%$ de la fréquentation totale pour les mois de mai à octobre (CDT des Landes, 2006a).

$\mathrm{Si}$, pour le référent surf de l'Union Nationale des Centres Sportifs de Plein Air (UCPA), la saisonnalité au sujet de son sport est de moins en moins effective : "la grosse saison, c'est sur 2 mois, mais il $\mathrm{y}$ a de plus en plus une avant et une après saison », il poursuit en affirmant que penser « l'initiation du surf uniquement en été, c'est réducteur ${ }^{7} »$. Pour autant, en 2006, les emplois salariés en CDI, tous au sein d'association, ne représentaient que $4 \%$ de l'ensemble des moniteurs de $\operatorname{surf}^{8}$ (CDT des Landes 2006b). Les 96 \% restant étaient distribués au sein des associations et des entreprises comme suit : CDD : $66 \%$; travailleurs indépendants : $20 \%$; gérants non salariés : $10 \%$. Ces deux dernières catégories sont en outre quasi-exclusivement concernées par la saisonnalité aux dépens d'emplois annualisés. Ainsi, beaucoup de moniteurs de surf, principale activité nautique pourvoyeuse d'emplois dans les Landes, ne restent pas jusqu'à cinq ans d'ancienneté dans l'association ou l'entreprise qui les emploie et la plupart considèrent les niveaux de rémunération comme étant trop faibles. Il en résulte, selon Robert Castel (1995), que «l'insertion n'est plus une étape, elle demeure un état ».

À la lumière de ces données sur le tourisme et les sports nautiques, il n'est pas incongru de dire que les effets de la saisonnalité landaise sont si structurants qu'ils empêchent, quasi-mécaniquement, de développer des activités associatives et commerciales dans les sports nautiques tout au long de l'année (Guibert, Slimani, 2011). Il paraît alors difficile voire impossible, en dehors des périodes de fortes affluences touristiques, de penser le marché de l'emploi, en particulier sportif, capable d'absorber le reflux de travailleurs saisonniers qui se retrouvent sans employeur. Ainsi, le territoire des Landes se caractérise par une alternance entre "le vide » et " le plein », sur le modèle des vases communicants d'une économie locale marquée, en termes de structure d'emplois, par une saisonnalité touristique dense, une offre d'animation sportive par les activités nautiques relativement pourvue et très structurée par la domination du surf et l'investissement

7. Entretien effectué auprès du référent surf de l'UCPA, juin 2008. 8 . Ils sont 240 au total. 
des élus locaux qui en font un vecteur de développement de leur politique territoriale. Finalement, les offres des écoles d'activités nautiques étant très largement axées vers de la découverte et de l'initiation à destination d'une clientèle touristique, les postes offerts ne peuvent qu'être en grande majorité des CDD de périodes plutôt courtes synchroniques à la saisonnalité. Les salaires pratiqués dans les associations sportives sont, par ailleurs, généralement inférieurs à ceux pratiqués dans les écoles commerciales. Souvent, en saison, dans les associations, le moniteur permanent est moins rémunéré (1600 € net par mois en moyenne) que le moniteur en CDD (2 $000 €$ net par mois en moyenne). Le niveau de rémunération varie en sens inverse du niveau hiérarchique de responsabilités. Les gérants d'entreprises commerciales et leurs employés touchent les salaires les plus élevés sur un laps de
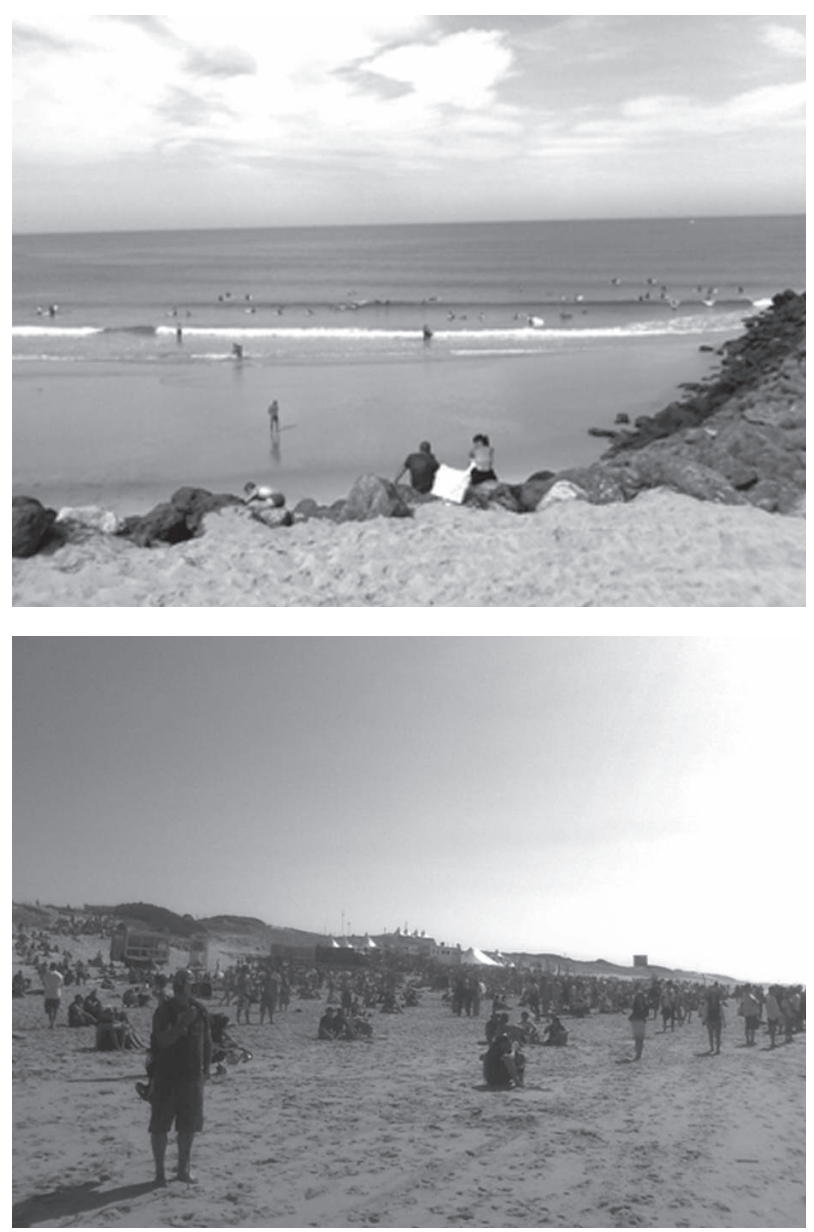

temps plus court (Guibert, 2008). Ces variations de rémunérations incitent des moniteurs à travailler en CDD plutôt qu'en CDI, en entreprises plutôt qu'en association, en saison estivale plutôt qu'à l'année. Partant, des effets imprévus liés à la concentration de pratiquants au sein des lieux de pratique puis à la concurrence territoriale des espaces se manifestent étant donné que le « gâteau à se partager » n'est pas spatiotemporellement extensible.

\section{UN LITTORAL VICTIME DE SON SUCCÈS : CONCENTRATIONS, CONCURRENCES ET CONFLITS}

\section{Un espace littoral convoité mais limité}

Le littoral landais est un des moins densément peuplé des littoraux français. De nombreux lacs, des
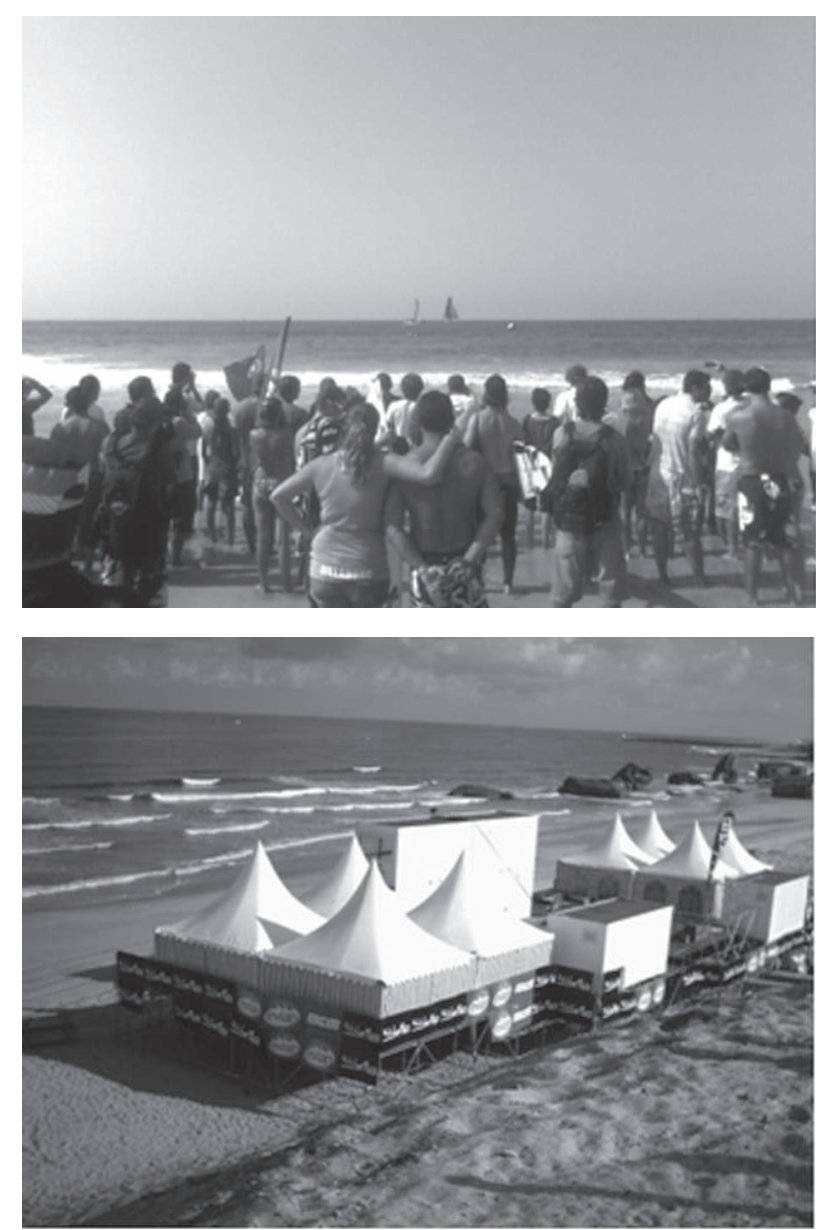

Figures 7, 8, 9, 10 : Quand le surf monopolise l'espace public (cliché de l'auteur) When surfing is monopolizing the public space 
zones naturelles protégées, un espace dédié à une zone militaire (à Mimizan et Biscarrosse), les dunes puis la forêt font que les accès au littoral sont très localisés et délimités. De ce "patrimoine naturel original fragile» (Larroque Chounet, 1998) résulte une concentration urbanistique dense mais localisée sur le littoral d'une part et une agrégation touristique d'autre part due à un immobilier de loisirs également très circonscrit : le linéaire côtier landais « mis en tourisme demeure peu étendu » (Duhamel, Violier, 2009). En conséquence, les effets de concentration des pratiquants d'activités nautiques dans les sites et espaces se font très prégnants malgré un linéaire côtier homogène (la plage). Que ce soit la privatisation visuelle, symbolique, mais aussi spatiale par les écoles ou par les compétitions professionnelles de surf, la communication quasiexclusive de collectivités locales à l'égard des sports nautiques, les clichés proposés ici (figures 7 à 9) témoignent du caractère incontournable du surf sur le littoral des Landes et, plus globalement, de la littoralisation des pratiques (Delfaud, 2005) et de ses effets imprévus en termes de conflits d'usages potentiels.

\section{Des activités sportives concurrentielles sur les lieux de pratique}

Les spécificités mêmes liées à la pratique des activités nautiques engagent des effets non désirables. Si le jet ski peut se pratiquer en haute mer, il n'est pas rare de voir des pratiquants aller dans les vagues près du rivage. Les pêcheurs à la ligne en bord de mer (surf-casting) recherchent quant à eux les zones où les vagues déferlent, plus propices à la pêche, mais qui sont également les espaces de jeu des surfeurs et bodyboardeurs... Cette situation soulève finalement la question de l'usage légitime des espaces littoraux et des logiques sociales d'appropriation de l'espace. Les conflits d'usage, en l'absence de médiateur et très peu relayés par la presse locale - à l'inverse de conflits locaux parfois très médiatisés (Torre, Lefranc, 2006) - impliquent dans le département des Landes des régulations pour le moins strictes de la part des municipalités les plus concernées.

\section{Jet ski, pêche en mer et kite-surf: des activités spatialement concurrencées}

Les zones de «mise à l'eau » des jets ski en milieu marin sont quantitativement et spatialement réduites : il n'existe qu'un seul port maritime dans les Landes, situé à Capbreton. À Mimizan, l'embarquement se réalise dans le courant d'Huchet. Le bruit et la pollution des jets puis la sécurité sont parfois décriés par les résidents locaux même si aucun accident n'est à déplorer selon le gérant d'une entreprise de location de jet ski située à Capbreton. Les comportements des pratiquants qui louent le matériel (donc sans moniteur accompagnateur) sont parfois peu respectueux des règlements maritimes en vigueur (les « 300 mètres », la vitesse maximale autorisée dans le port, etc.) concourant à la détérioration de l'image du jet ski. Il en résulte des politiques municipales limitatives : suite à des abus de pratiquants, la municipalité de Biscarrosse a borné strictement la pratique du jet ski du lundi au jeudi, de 15 heures à 19 heures uniquement (des panneaux d'interdiction sont disséminés à l'abord des sites de pratique). "On galère, c'est dommage: on a récolté les abus des particuliers qui ne respectaient rien! » précise un moniteur de jet ski, salarié d'une entreprise de la commune qui, suite à cette décision municipale, "a du partir [de l'entreprise] car ce n'était plus rentable». Pour autant, le propos de la Directrice de l'ODT de Biscarrosse témoigne d'une situation pour le moins dangereuse : "Il y a eu un ras le bol: il y a en a qui ont fait les cons, qui partent dans la zone des 300 mètres avec des plongeurs dessous!» Elle indique toutefois que le débat est davantage électoraliste que sur les réels désagréments de ce sport (bruit, danger, etc.) : «Oui, c'est pour ceux qui font la sieste l'après-midi et qui ne veulent pas être dérangés. C'est politique, c'est sûr. »

"On est banni des plages à touristes l'été, des plages à activités nautiques. Ce sont les joyeusetés de la gestion des plages! »C'est en ces mots que le Président du CD de pêche en mer explique, quant à lui, la situation relative au partage des espaces balnéaires. Souvent obligés de s'éloigner des zones d'activités (baignade et surf principalement), les pêcheurs au " surf casting » ou au « lancé-ramené » ne peuvent choisir leurs sites de pêche indépendamment des autres pratiquants. En été, les horaires privilégiés de pêche, le matin tôt, permettent par ailleurs aux pêcheurs d'éviter la surfréquentation de certaines plages. Pour ce pêcheur retraité, propriétaire d'une résidence depuis une vingtaine d'années à Capbreton, tout est question de compréhension des usages des autres pratiquants (notamment les 
surfeurs et les jet skieurs) puis du respect des règles afin d'éviter ou de minimiser les tensions sousjacentes :

"C'est vrai que parfois c'est vraiment gênant. On est obligé de s'éloigner. Mais souvent, les surfeurs viennent, ils arrivent sur la plage et ils voient les cannes. Certains me demandent comment sont les courants pour ne pas gêner, d'autres s'éloignent. Par contre, ça arrive qu'il y en ait, c'est des surfeurs débutants qui ne connaissent ni les vagues ni les courants, qui foncent sans regarder et là, je les engueule! La plupart viennent s'excuser... Ensuite, il y a les jet ski : ils sont quatre ou cinq, toujours les mêmes, et ils viennent dans les vagues, près $d u$ bord, là où on pêche. Donc les 300 mètres, ils ne respectent pas : ils s'en foutent ${ }^{9}$ ! »

Concernant le kite-surf, les améliorations technologiques et techniques en font une activité au développement quantitatif constant depuis plusieurs années. Il en résulte des phénomènes de surfréquentation des spots identifiés lorsque les conditions météorologiques sont jugées bonnes. La création récente d'une école de kite (entreprise commerciale) à Biscarrosse témoigne de l'existence d'une demande. Son gérant est, selon ses propres termes, " sollicité chaque jour en été pour pouvoir pratiquer, que ce soit pour prendre un cour et découvrir l'activité ou tout simplement pour connaître les spots, c'est-àdire les lieux autorisés ». Ce dernier considère que la hausse du nombre de pratiquants d'année en année est de $20 \%$ à $30 \%$ : «Il y a une explosion de la pratique depuis deux ou trois ans avec le matériel qui est de moins en moins onéreux ». "Plein un ou deux mois à l'avance », les enseignements de kite-surf font recette : près de 180 prestations ont été vendues lors de la saison 2009, ce qui représente environ 60 clients, sachant que la moyenne est de trois cours par client. "La demande est telle que je refuse régulièrement des clients en été : des touristes mais aussi des gens de Mimizan, du Pays Basque, etc. » indique le propriétaire de l'école. Une réglementation stricte des pouvoirs publics réduisant les zones de pratique, en lac et en mer (en période estivale) est néanmoins de plus en plus effective. Les problèmes potentiels de sécurité sont au centre de ces préoccupations ${ }^{10}$.

9. Entretien effectué avec un pêcheur de Capbreton, septembre 2009.

10. La mort d'un kite-surfeur à Saint-Jean-de-Luz, début novembre 2010, renforce ce constat.
Au-delà d'une tarification socialement sélective ${ }^{11}$, davantage que pour un stage de voile ou de surf, d'autres freins sont recensés par le président du club de Biscarrosse, notamment le manque, de plus en plus sensible, d'espaces de pratique. Une seule zone est actuellement autorisée sur le lac par la municipalité de Biscarrosse. De nombreuses autres zones autour du lac sont inaccessibles pour se rendre sur site, d'autres sont protégées et classées « Natura 2000 », ce qui exclut toute activité nautique. Il en résulte, mécaniquement, "une surpopulation qui va arriver très prochainement » selon le président du club local. Pour ce dernier, la municipalité de Biscarrosse n'est, par ailleurs, "pas très réceptive : il ne considère pas le kite-surf comme un produit touristique».

\section{Le surf : l'activité qui cristallise les rivalités en période estivale}

Le surf dans le département des Landes est une activité nautique incontournable. Que ce soit sur le plan des effectifs licenciés, du nombre d'associations ou d'entreprises, de l'emploi des moniteurs, de l'impact touristique ${ }^{12}$ ou encore des logiques d'identification territoriale, le surf est l'activité nautique phare. C'est sur le littoral landais que la concentration de clubs et surtout d'écoles commerciales (entreprises) en période estivale est la plus prégnante (figure 11). La seule commune de Seignosse compte en effet, lors des étés 2008 et 2009, près d'une quinzaine d'établissements, très majoritairement de type entrepreneurial. «Il y a environ 50 à 60 entreprises qui donnent des cours de surf, pour une quinzaine de clubs dans les Landes. Ça fait une manne d'emplois énorme. Certaines entreprises n'ouvrent que deux mois, parfois trois mois ${ }^{13}$ " indique le Président du Comité départemental de surf des Landes. Sur le site Internet de la FFS ${ }^{14}$, 42 écoles labellisées sont comptabilisées. Ces dif-

11. Le tarif pratiqué pour une séance de 3 heures (matériel compris) s'élève ordinairement à $100 €$.

12. Selon les déclarations des répondants à un questionnaire passé à la clientèle des écoles de surf (enquête 2008), près de la moitié des individus de l'échantillon $(49 \%)$ affirme que la volonté de pratiquer le surf a eu un impact sur le choix du lieu de vacances d'été.

13. Entretien effectué auprès du Président du Comité départemental de surf des Landes, septembre 2009. Ce dernier, gérant d'entreprise, est également Président du Capbreton Surf Club et vice-président de la Ligue de surf d'Aquitaine. Il occupe une position élevée dans l'espace du surf associatif français.

14. Source: www.surfingfrance.com, consulté le 25 novembre 2010. 
férences d'appréciation proviennent du fait qu'un bon nombre d'écoles sont créées ou résiliées chaque année, que certaines écoles sont "multiactivités » (voile, surf, canoë, etc.) et brouillent l'identification, ou encore que certaines sont adossées à un surfshop ou un shaper et que l'activité n'est, dans ce cas, pas systématiquement reconduite chaque année. Enfin, la labellisation des écoles facilite la quantification de ces dernières. Autrement dit, d'une année à l'autre, il est difficile de définir et prévoir le nombre exact d'écoles de surf. Cette "flexibilité quantitative » interroge les élus locaux des communes littorales pour lesquels il est difficile d'appréhender sur le long terme le développement de l'offre de l'enseignement du surf, d'autant plus que se surajoutent les écoles étrangères qui viennent, en été, profiter du climat et de la qualité des vagues du littoral landais.

L'attrait de la côte landaise pour la pratique du surf dépasse les frontières nationales, que ce soit pour les pratiquants chevronnés ou les débutants. Plusieurs dizaines d'écoles commerciales étrangères (allemandes et anglaises principalement) dispensent ainsi des enseignements aux pratiquants étrangers. Ces derniers achètent des séjours dans leurs pays respectifs (surf et hébergement généralement) et suivent les cours et les stages sur les plages du littoral français. Si la liberté de travailler et l'utilisation de l'espace littoral par les moniteurs étrangers ne sont pas remises en cause par les moniteurs et dirigeants français ${ }^{15}$, plusieurs problèmes sont néanmoins soulevés par ces derniers, illustrant les concurrences entre les écoles et un sentiment « d'invasion ». Les responsables d'écoles qui ont évoqué, dans le cadre des entretiens, la question des écoles étrangères témoignent des carences aussi bien techniques que sécuritaires des moniteurs de surf étrangers. Selon eux - et sans que ces arguments soient vérifiés par les services de l'Etat compétant en la matière -, les règles de sécurité ne sont pas toujours respectées : nombre conséquent d'élèves par moniteur, sélection de la zone de pratique peu appropriée, concentration de groupes sur un espace restreint. Egalement, la pédagogie mise en œuvre est qualifiée de "basique » et peu optimale (moniteur sur la plage plutôt que dans l'eau avec les élèves, « animation » plutôt qu'initiation, etc.).

Plusieurs responsables français d'écoles de surf indiquent en effet que la British Surfing Association (BSA) dispense des formations très différentes du BPJEPS. Le « Level 1 », premier niveau permet-

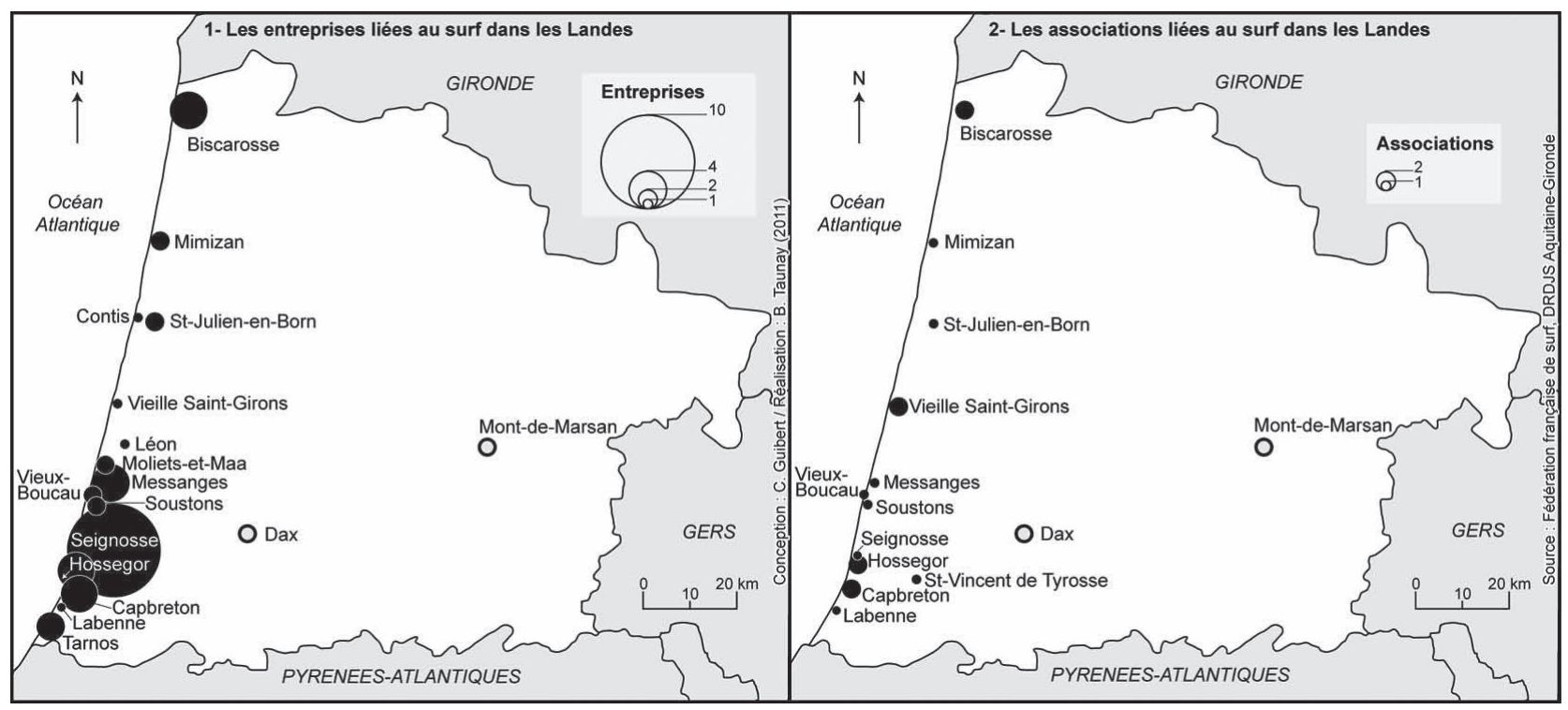

Figure 11 : Le surf dans les Landes : une concentration dans le sud du littoral du département Surfing in the Landes: a south coast of the department concentration

15. Des moniteurs de surf français assurent toutefois des cours de surf auprès de Français dans des pays étrangers (Maroc, Portugal, etc.). 
tant d'enseigner à des débutants peut être validé en trois jours, moyennant 250 livres (soit environ 300 à 350 euros $)^{16}$. Les pré-requis sont par ailleurs peu exigeants : un diplôme de sauvetage homologué puis deux ans de pratique personnelle du surf (sans que le niveau technique soit précisé). Ce décalage entre les formations anglaises et françaises en termes de pré-requis techniques et de sécurité puis en termes de formation est vivement critiqué par la plupart des interviewés. Il convient par exemple d'avoir a minima un niveau de pratique régional en France, puis de valider un diplôme d'Etat (BPJEPS) dont la formation dure soit 350 heures (parcours « court » dispensé par la FFS si le stagiaire possède déjà des brevets fédéraux et est compétiteur affirmé) soit 840 heures (parcours « long » de 12 mois, dispensé par l'UCPA Formation en l'absence des formations et niveaux exigés pour le parcours « court»). Aussi, les critères définis par les services Jeunesse et Sports permettant ou non à une école étrangère d'assurer des prestations en France sont-ils mis en cause selon ces responsables interrogés en 2008 (Guibert, 2008) :

"Il y a une invasion d'écoles étrangères, ça peut même devenir conflictuel. Les critères de Jeunesse et Sports sont à des années lumières des exigences en France. C'est une honte. » (Moniteur d'une association, 27 ans.)

«La DDJS ne fait pas assez de contrôles des accréditations temporaires et occasionnelles. Les surfcamps étrangers ne paient pas les mêmes charges que nous: on ne peut pas rivaliser. En plus, on n'a pas le droit d'employer un BSA Level 1 en France! » (Gérant d'une entreprise, 32 ans.)

"Il y a un flou juridique et un décalage complet entre ce que fait la DDJS et la réalité! Nous, on est connu : mon école existe depuis 15 ans et c'est toujours nous qu'on contrôle! Alors que les écoles étrangères, elles, ça passe comme une lettre à la poste... » (Indépendant, 36 ans.)

On comprend alors pourquoi, à l'aune des fortes fréquentations spatialement localisées, des concurrences entre prestataires de services marchands et des conflits d'usages dans les espaces et les sites de pratique, des processus de régulation émanant des collectivités locales administrant les territoires soient de plus en plus effectifs.

16. Source : [http://www.britsurf.co.uk].

\section{LA Mise SUR AGENDA POLITIQUE DE L'ENSEIGNEMENT DU SURF : DES RÉGULATIONS LIMITATIVES}

La question de la concentration localisée des écoles de surf - relayée par la presse locale sans qu'il soit explicitement question de conflit- et plus précisément la densité des moniteurs de surf en période estivale sur des plages landaises bien circonscrites posent question aux élus locaux : «L'engouement de ce sport se vérifie aussi bien par des surfeurs individuels se mettant à l'eau sans encadrement, que par des groupes constitués dont les effectifs explosent. Un embouteillage côtier qui déferle désormais au mépris de règles de sécurité élémentaire. "On est victime de notre succès", confesse le maire de VieuxBoucau ${ }^{17}$. »Sur le plan technique, le littoral landais, très linéaire, offre peu de zones de replis. Seuls les spots de Capbreton autorisent par exemple la pratique du surf lors des tempêtes et grosses houles. Cette spécificité engendre un surpeuplement du littoral capbretonnais tout au long de l'année qui s'accroit en été : " "C'est un phénomène nouveau, amplifié chaque année. On peut donner des cours de surf sans forcément avoir de pied-à-terre", explique l'adjoint délégué aux sports de Capbreton. "Commençaient à poindre certains risques entre les baigneurs et les surfeurs", complète le maire, soulignant une certaine dérive mercantile ${ }^{18}$. »

À l'image des stations de montagnes alpines (Corneloup, Soulé, 2007), sécurité et partage cohérent des zones de loisirs (baignade, sports nautiques, etc.) sont à l'origine d'arrêtés municipaux visant à limiter le déploiement difficilement contrôlable des écoles de surf. Il s'agit soit de limiter le nombre d'écoles et donc de moniteurs en définissant des procédures de concessions (volonté de Biscarrosse), soit de limiter temporairement l'occupation du domaine maritime (Seignosse). Ces choix municipaux cadrent l'activité en la régulant sur le plan quantitatif. Cependant, des décisions trop strictes empêchent à l'activité professionnelle de moniteur de surf de se développer en été. Par ailleurs, selon certains responsables de club ou gérant d'entreprises, l'argument sécuritaire avancé par certaines municipalités est infondé vu le faible taux d'acci-

17. Sud-Ouest, "Les écoles de surf font des vagues à Vieux-Boucau », 16 juin 2010.

18. Sud-Ouest, « Moins d'apprentis surfeurs dans l'eau », 20 juillet 2010. 
dent au regard du nombre de pratiquants. Pour la Directrice de l'office de tourisme de Capbreton : « Ça peut être difficile, même avec les touristes... tout le monde voudrait avoir sa petite plage privée! Mais bon, c'est bien règlementé ici donc s'il y a de la concurrence, il y a une sorte d'émulation et c'est mieux. Il n'y a jamais de réclamation sur les écoles de surf, pas de mauvais retour donc c'est satisfaisant : je pense que ce sont des personnels sérieux. »

Les municipalités n'ont pas trouvé de solutions permettant une adéquation entre la gestion des plages (fréquence d'utilisation, espaces utilisés), le nombre d'écoles de surf et de moniteurs par école puis une optimisation de l'activité professionnelle des moniteurs de surf sur la durée d'une saison estivale. Le Directeur des Sports de la ville de Biscarrosse avoue être désarmé à l'égard de la situation en été sur les plages de la commune. En 2009, un club et cinq entreprises se sont partagées les enseignements de surf (le club est davantage polarisé sur les entraînements des jeunes du club). Trois autres demandes (des entreprises) ont été à l'étude pour pouvoir donner des cours de surf pour la saison 2010 :

« Je vais proposer à mes collègues de consulter un avocat. On a essayé de proposer un arrêté municipal l'an dernier pour organiser un peu les cinq écoles cet été. Mais là, on ne peut pas interdire à une école de venir s'implanter : on ne peut pas empêcher de travailler. Mais il y a quand même un problème de sécurité parce que les écoles, il y a parfois deux, trois et jusqu'à huit moniteurs (donc soixante-quatre pour une école). Donc moi je ne sais plus faire: on essaie de les réunir tous les ans, en plus ils essaient de garder un peu leur terrain et ils sont pas très enclin à voir d'autres écoles s'installer. La solution, je ne pense pas qu'elle existe. J'ai consulté mes collègues de la Côte Basque : ils ont procédé par concessions du domaine maritime... Chez nous, les Affaires Maritimes de Capbreton m'ont dit que ce n'est pas possible donc moi, ça m’a bloqué! Je m'étais appuyé sur un document de Biarritz (le collègue qui est devenu DTN à la fédé) mais on m'a dit non: le gars de Capbreton m'a dit que je ne pouvais pas interdire une école itinérante de donner des cours de surf et qu'il allait me faire payer très cher les concessions du domaine maritime. Donc là, il y a un problème: c'est une épine qu'on a dans le pied et c'est vraiment problématique. Ma proposition, ce serait plutôt de limiter à deux le nombre de moniteurs par école en donnant la possibilité à plus d'écoles de s'implanter ${ }^{19}$. "

Le dilemme est le même pour la commune de Capbreton qui, de par une situation abritée sur le littoral grâce aux jetées du port, voit le nombre d'écoles augmenter lorsque les conditions météorologiques empêchent l'initiation au surf sur les plages des communes voisines :

"La vérité d'un site n'est pas forcément celle de l'autre. On a une commission en janvier avec des élus, le Service des Sports et des membres de la fédération. Donc tout le monde ne sera pas content... Globalement, ce sera de réduire le nombre de moniteurs. Cet été, sur Capbreton il y avait sept / huit écoles, sans compter celles de Seignosse ou Labenne qui viennent ici quand les conditions de mer ne sont pas bonnes par ce que c'est accessible. Donc il y a un souci et c'est surtout pour la sécurité... c'est un gros chantier pour la commune ${ }^{20}$. »

Le problème engagé par les procédures municipales en période estivale réside dans l'impossibilité de sélectionner le lieu de pratique en fonction des conditions météorologiques, de vagues ou de marées. En effet, selon le coefficient de marée et l'heure de la journée par exemple, un spot sera plus propice qu'un autre pour enseigner le surf à des débutants. Or, cette conséquence est en contradiction avec un des critères de la délivrance du label fédéral « Ecole française de surf » puisque le « Choix du site [doit être] adapté au niveau des élèves et des conditions de mer $^{21}$ ». Les arrêtés municipaux interdisent donc les écoles labellisées de répondre à la totalité des critères du dispositif. Autrement dit, les limitations (du nombre d'école et/ou de moniteurs par école) sont globalement vécues comme une contrainte qui n'optimise pas la qualité de l'enseignement même s'il réduit la surpopulation des espaces et sites de pratique. Pour ce moniteur-gérant d'une école de surf de la commune de Labenne (31 ans, ancien compétiteur de haut niveau), les contraintes municipales « ne permettent pas toujours de répondre aux attentes des clients qui veulent changer de site si les vagues sont trop grosses ici ». Dans le cas de la ville de Seignosse,

19. Entretien effectué avec le Directeur des Sports de la ville de Biscarrosse, novembre 2009

20. Entretien effectué avec le Directeur des Sports de la ville de Capbreton, novembre 2009

21. Source : Charte de qualité du label fédéral délivré par la FFS. 
la seule contrainte a été d'interdire toute utilisation du domaine maritime pour établir des cabanes à partir de mi-septembre. Pour un gérant de structure commerciale (37 ans, gérant depuis 7 ans), « ça constitue un frein puisque l'activité peut potentiellement continuer jusquà fin septembre ${ }^{22}$ ».

Le Comité Départemental (CD) de surf est très concerné par cette problématique et notamment sur les conséquences des arrêtés municipaux tels qu'ils existent actuellement. Une piste proposée par le Président du CD consiste à établir un forfait à l'égard des entreprises souhaitant offrir des cours de surf, lequel permettrait aux municipalités de subventionner les associations afin de pérenniser les emplois :

«On a commencé à y réfléchir! C'est que les écoles privées paient une sorte de patente, qu'ils aient une plage l'été moyennant quoi cette patente serait reversée au club local. Anquel cas, le club, lui, arrête d'être commercial et d'être concurrentiel des écoles privées. C'est une solution aussi... qui permettrait de désengorger les plages. Cet été, on a comptabilisé : plus que le nombre d'école, c'est le nombre de salariés qui se retrouvent à l'eau à donner des cours. On a compté parfois 18 ou 20 BE sur Capbreton et lorsqu'il n'y a pas assez ou à l'inverse trop de vagues, ils se retrouvent tous sur la même plage! Donc 18 groupes de 8 élèves, c'est ingérable avec les zones de baignade et les free surfeurs! Aujourd'hui il faut limiter le nombre de BE par école et le nombre d'école... Mais c'est vrai que ce n'est pas très cool pour les mecs qui veulent se développer. Mais à un moment... Ça engage des questions de sécurité, de qualité de l'enseignement... il y a plusieurs dimensions qu'il faut prendre en compte! »

Ce dispositif visant à dissocier les offres des associations de celles des entreprises permettrait de réduire la concurrence qualifiée de déloyale par certains gérants (les locaux des associations sont souvent mis gracieusement à disposition, hors les prestations des associations sont similaires à celles des entreprises). Par ailleurs, si une entreprise commerciale estime être victime localement de concurrence déloyale de la part d'associations, elle peut engager une action contentieuse devant le juge civil sur la base des articles 1382 et 1383 du Code civil. Il importe dans ce cas qu'elle puisse démontrer la

22. Entretien effectué auprès d'un gérant d'école de surf de Seignosse, juillet 2008 . réalité du dommage à son activité (baisse du chiffre d'affaires par exemple) et le lien de causalité entre l'activité de cette ou ces associations et la baisse du chiffre d'affaires en résultant pour son entreprise.

Si les sports nautiques touristiques peuvent s'envisager comme de véritables « ressources territoriales » (Gumuchian et Pecqueur, 2004) par les élus locaux, force est de constater que certains effets, dont les ampleurs sont souvent mal mesurées, engagent des limitations de manière croissante. C'est en quelque sorte, pour certaines municipalités, la « rançon de la gloire » suite aux marquages territoriaux structurés par les compétitions professionnelles de surf notamment (Capbreton, Hossegor, Seignosse). Les régulations quantitatives et/ou spatialisées de certaines modalités de pratique ont toutefois, on l'a vu, des répercussions sur l'économie d'un secteur d'emplois, celui des moniteurs sportifs.

\section{Conclusion}

Les effets de la saisonnalité touristique et les spécificités spatiales sont, sur le littoral des Landes, si prépondérants qu'ils empêchent de développer continument une activité professionnelle liée à la découverte et l'initiation des sports nautiques en été, particulièrement en surf. Il risque de devenir, par conséquent, difficile à terme, à l'appui de ces données, de «fournir » une activité professionnelle à chacun des moniteurs sportifs formés et diplômés. Déjà, au sein de certaines communes des PyrénéesAtlantiques - ou les espaces sont plus réduits que dans les Landes -, le nombre de moniteurs de surf est limité de manière stricte. Il s'agit là bien de conflits qui renvoient directement à la « question d'affectations des espaces » (Mormont, 2006) par les municipalités à l'égard des différents acteurs du secteur des services sportifs. Si ce type de régulations se généralise à l'ensemble des plages landaises, les offres d'emploi se feront sans doute de plus en plus rares. Sans vouloir « à tout prix » penser en termes d'adéquation "formations/emplois », les sources potentielles d'emplois se situeront hors saison, que ce soit dans le domaine des activités sportives (des offres touristiques seront à identifier) ou dans d'autres secteurs d'activités professionnelles.

La dimension territoriale des «système activités professionnelles atypiques » (Perrin-Sanchis, et al., 1997) n'est pas prise en compte par les instances en 
charge de l'emploi sportif dans le département des Landes. Aussi, produit touristique par excellence entretenant un "rapport enchanté au monde » (Réau et Poupeau, 2007), les activités nautiques sur le littoral landais n'en constituent pas moins une offre qui s'inscrit dans un rapport marchand où le « donnant-donnant » n'est pas toujours de mise. Les profits symboliques et économiques engendrés par le département des Landes, en particulier les communes littorales comme en témoignent les stratégies affirmées d'identification territoriale grâce à « la plage » et aux activités dites de «glisse » selon le CDT, n'équivalent pas les avantages que peuvent espérer en attendre, professionnellement parlant, les moniteurs sportifs.

\section{Bibliographie}

Augustin J.-P., 1994. « Le littoral aquitain, de la station balnéaire à la station surf », Surf Atlantique, Les territoires de l'éphémère, Bordeaux, MSHA, 272 p.

Bavoux J.-J., Bavoux D., 1998. Géographie humaine des littoraux maritimes, Paris, Armand Colin, 95 p.

Castel R., 1995. Les métamorphoses de la question sociale, Paris, Fayard, 813 p.

Comité Départemental de Tourisme des Landes, 2003, 2008. « La clientèle séjournant sur le littoral landais ».

Comité Départemental de Tourisme des Landes, 2006 a. «Activités sportives et de loisirs », 8 p.

Comité Départemental de Tourisme des Landes, 2006 b. « La filière surf », 33 p.

Comité Départemental de Tourisme des Landes, 2007. «Les chiffres clés du tourisme dans les Landes », 19 p.

Comité Régional de Tourisme d’Aquitaine, Observatoire RÉGional du tourisme en Aquitaine, 2005, « Les chiffres clés du tourisme en Aquitaine ».

Corneloup J., Soule B., 2007. « La gouvernance sécuritaire dans les stations de sports d'hiver françaises », Espaces et sociétés, $\mathrm{n}^{\circ}$ 128-129, p. 133-150.

Chazaud P., 2004. Management du tourisme et des loisirs sportifs de pleine nature, Voiron, PUS, $350 \mathrm{p}$.

Delfaud P., 2002. «La région, chef de file du développement économique en Aquitaine », in Hudon R., Augustin J.-P., Villes, régions et universités. Recherches, innovations et territoires, Presses universitaires de Laval, p. 163-181.

Direction Régionale de la Jeunesse et des Sports PACA, 2009. Les métiers des sports de montagne, évolution et enjeux en PACA, $126 \mathrm{p}$.

Duceux Y., 2008. «Educateur sportif voile : du saisonnier au transitoire », Agora Débats/Jeunesse, n 47.

Duhamel P., Violier P., 2009. Tourisme et littoral. Un enjeu du monde, Paris, Belin, 191 p.

Guibert C., 2002. «Le sous-secteur surf », L'emploi sportif en France, situations et tendances d'évolution, AFRAPSRUNOPES, p. 115-117.
Guibert C., 2006. L'univers du surf et stratégies politiques en Aquitaine, Paris, L'Harmattan, 321 p.

Guibert C., 2008. Le surf en France : Emplois, employabilité et formations, étude commanditée par la DRDJS AquitaineGironde, 178 p.

Guibert C. et al., 2010. Etat des lieux des activités nautiques et de loisirs touristiques dans le département des Landes, étude commanditée par le CREPS d'Aquitaine, la DRJS d'Aquitaine et la DDJS des Landes, 252 p.

Guibert C., Slimani H., 2011. Emplois sportifs et saisonnalités. L'économie des activités nautiques : enjeux de cohésion sociale, Paris, L'Harmattan, 237 p.

Gumuchian H., Pecqueur B., 2004. « La notion de ressource territoriale », Montagnes Méditerranéennes, n²0, p. 4.

INSEE, 2009. « L'emploi salarié touristique double entre janvier et août », Les quatre pages INSEE Aquitaine, $\mathrm{n}^{\circ} 185$.

Larroque Chounet L., 1998. «Paysages et urbanisation sur la côte sud des Landes ", Sud-Ouest européen, n 1, p. 7-19.

Le Roux N., 2003. «Introduction générale », L'emploi sportif en France : situation et tendances d'évolution, AFRAPSRUNOPES, p. 9-16.

Marchais E., 1994. "Surf in Biarritz, une histoire d'amour qui fait des vagues », Cahiers Espaces, « Loisirs nautiques et aquatiques ", n³5, p. 108-115.

Mollard A., Pecqueur B., 2003. «Valoriser les qualités spécifiques des territoires : le panier de biens des Baronnies », Territoires, acteurs et agriculture en Rhône-Alpes, $\mathrm{n}^{\circ} 2$, novembre, $4 \mathrm{p}$.

Mormont M., 2006. «Conflit et territorialisation », Géographie, économie, société, Vol. 8, p. 299-318.

Paugam S., 2001. Le salarié de la précarité, Paris, PUF, 437 p.

Paugam S., 2009. La disqualification sociale : essai sur la nowvelle pauvreté, Paris, PUF, $288 \mathrm{p}$.

Perrin-Sanchis T., Perret J., Gerbaux F., 1997. « Pluriactivité et saisonnalité. Des atouts pour le développement des territoires ", Ingénieries EAT, n 12, p. 49-58.

Reau B., Poupeau F., 2007. «L'enchantement du monde touristique », Actes de la recherche en sciences sociales, Nouvelles (?) frontières du tourisme, $\mathrm{n}^{\circ} 170$, p. 5-14.

Sanchez P., 2007. Le surf en Aquitaine. Approche d'une discipline phare de la région, DRDJS Aquitaine, MAP, non paginé.

SIAU V., 2004. «Pour une approche territoriale des enjeux liés à l'emploi et à la formation ", Sports de nature. Des territoires et des hommes, Cahiers Espaces, n 82, p. 36-43.

SLimani H., 2003. Formation et stratégies de professionnalisation dans le secteur des activités équestres. Balise $n^{\circ} 2$, Sport et animation/Emploi et formation en Pays de la Loire, Conseil Régional, DRDJS et CARIF-OREF des Pays de la Loire, 4 p.

Sobry C., 2004. « Le tourisme sportif : de quoi parle-t-on? », Le tourisme sportif, Presses universitaires du Septentrion, p. 13-30.

Torre A., Lefranc C., 2006. "Les conflits dans les zones rurales et périurbaines. Premières analyses de la presse quotidienne régionale », Espaces et Sociétés, n 124-125, p. 93-110. 